\title{
Searches for ultra-high energy photons at the Pierre Auger Observatory
}

\author{
Philip Ruehl $^{* a}$ for the Pierre Auger Collaboration ${ }^{b}$ \\ ${ }^{a}$ University of Siegen, Department of Physics, 57068 Siegen, Germany \\ ${ }^{b}$ Observatorio Pierre Auger, Av. San Martín Norte 304, 5613 Malargüe, Argentina \\ E-mail: ruehl@hep.physik.uni-siegen.de \\ Full author list: http://www.auger.org/archive/authors_2019_03.htmI
}

\begin{abstract}
One of the key challenges in astroparticle physics is identifying the sources of cosmic rays at the highest energies (above $10^{18} \mathrm{eV}$ ). In this context, the search for ultra-high energy photons is of high interest. Observing photons of such energies would impact astrophysics and particle physics as well as fundamental physics and would be an important contribution to multimessenger astronomy. The Pierre Auger Observatory near Malarguie, Argentina, is the largest air-shower experiment for the detection of ultra-high energy cosmic rays. It consists of an array of about 1660 water Cherenkov detectors arranged on a triangular grid which covers an area of more than $3000 \mathrm{~km}^{2}$. Additional 27 fluorescence telescopes at four sites overlooking the detector array provide an independent and complementary method for air-shower detection.

In the contribution, the various activities at the Pierre Auger Observatory concerning searches for ultra-high energy photons will be presented, and the current results will be summarized.
\end{abstract}

The New Era of Multi-Messenger Astrophysics - Asterics2019

25 - 29 March, 2019

Groningen, The Netherlands

${ }^{*}$ Speaker. 


\section{Introduction}

In order to probe the origin and the interactions of cosmic rays at the highest energies, the search for photons with energies in the EeV range and above is one of the key challenges in astroparticle physics. Unlike charged cosmic rays, ultra-high energy (UHE) photons are not deflected by interstellar magnetic fields. This and their property of traveling at the speed of light makes them ideal candidates for multimessenger astronomy since their arrival time and direction directly pinpoint their origin in space. The flux of particles at energies above $\mathrm{EeV}$ is too low to allow for direct measurements of those particles in balloon or satellite-based experiments. Thus the secondary particles that are produced in extensive air showers (EAS) when the primary particles interact with the atmospheric molecules have to be measured in ground-based detectors.

Thanks to a surface detector (SD) array of 1660 water-Cherenkov detectors on an area of $3000 \mathrm{~km}^{2}$ and 27 fluorescence detectors (FD) overlooking the SD array, the Pierre Auger Observatory [1] has a unique exposure to UHE photons. The complementary detection techniques of the two detector types allow for discrimination between primary photons and hadronic particles. In this contribution, the integral upper limits on the diffuse photon flux above $1 \mathrm{EeV}$ obtained from data measured with both, the SD alone and the combination of the SD and the FD (hybrid detector) will be reported together with the results of a dedicated search for point sources of UHE photons.

\section{Search for a diffuse photon flux}

The search for a diffuse flux of UHE photons is separated in two analyses, one using data from the SD and one with hybrid data. While the hybrid detector allows for a strong separation between photon and hadron induced air showers, its duty cycle is bound to the operability of the FD, which is limited to clear and moonless nights. The SD is independent of weather conditions and thus has a duty cycle of almost $100 \%$.

\subsection{Photon search with the SD}

In the photon search with the SD [2], two observables have been forged to separate photon showers from the hadronic background. The first observable, $L_{L D F}=\log _{10}\left(\frac{1}{N} \sum_{i} \frac{S_{i}}{L D F\left(r_{i}\right)}\right)$ makes use of the steeper profile of the lateral particle distribution (lateral distribution function, LDF) in a photon induced shower compared to the average data event. The LDF can be measured with the SD by looking at the signal $S_{i}$ in an SD station at a distance $r_{i}$ from the shower axis. In the definition of $L_{L D F}$, the signal $S_{i}$ of the $i$-th triggered SD station in the event is divided by the average data LDF at distance $r_{i}$ and summed over all $N$ stations in the event. For an average data event, $L_{L D F}$ is expected to be close to 0 . Thus, $L_{L D F}$ for photon-induced showers is expected to be negative. The second observable, $\Delta$ is given by $\Delta=\frac{1}{N} \sum_{i} \delta_{i}$ with $\delta_{i}=\frac{t_{1 / 2}^{i}-t_{1 / 2}^{\text {Bench }}}{\sigma_{t_{1 / 2}}^{i}}$ being the residual of the signal rise

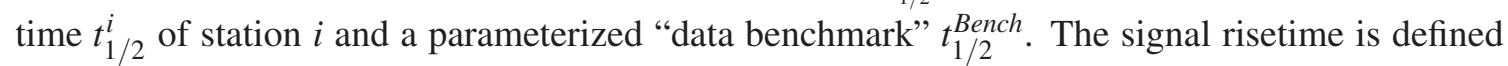
as the time difference between the $10 \%$ and $50 \%$ quantiles of the SD signal traces. $\sigma_{t_{1 / 2}^{i}}$ is the expected width of the distribution of $t_{1 / 2}$. Since an abundance of muons in an air shower causes a short risetime, photon showers are expected to produce large values of $\Delta$. The separation power of $L_{L D F}$ and $\Delta$ is combined in a principal component (PC) analysis (Fig. 1). The final cut is placed on 
the PC at the median of the distribution of non-preshowering [3] photon events. Events with a PC larger than the cut value are considered photon candidate events. The analysis has been performed separately, above $10 \mathrm{EeV}, 20 \mathrm{EeV}$ and $40 \mathrm{EeV}$ and applied to data collected between January 2004 and May 2013. The number of photon candidate events found in the respective ranges were 4, 2 and 0 . Since these results are consistent with the expectation from the hadronic background, upper limits at 95\% C.L. have been placed on the integral photon flux (Fig. 3).
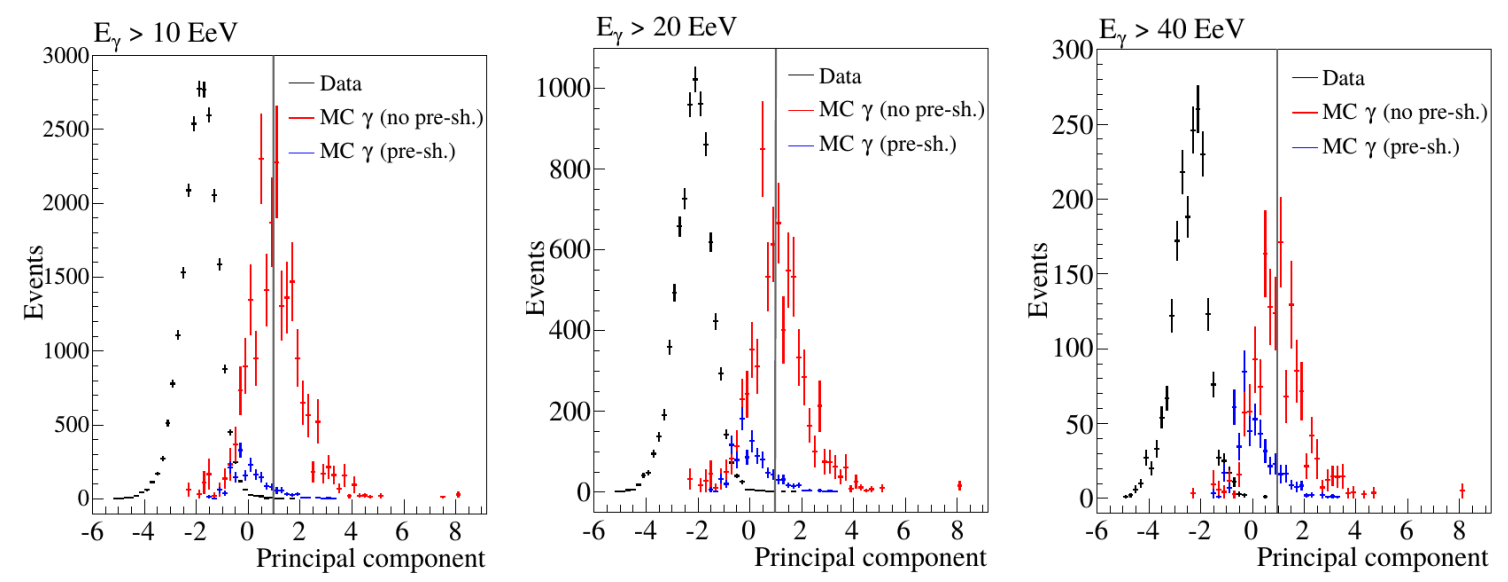

Figure 1: The principal component of the two photon observables $L_{L D F}$ and $\Delta$ for data (black) and simulated photon showers (blue for preshowering and red for non-preshowering photon primaries). The photon candidate cut on the principal component lies at the median of the distribution of the non-preshowering photon events and is indicated by the vertical line for each of the three energy ranges. [2]

\subsection{Photon search with the hybrid detector}

For the photon search with the hybrid detector [4], two photon observables were used which take advantage of the complementary data collected by the SD and the FD. The observable $X_{\max }$ is the atmospheric depth of the longitudinal shower maximum and can be directly observed with the FD. Photon showers are expected to have an $X_{\max }$ which is on average about $100-150 \mathrm{~g} \mathrm{~cm}^{-2}$ deeper than a typical hadronic shower. The observable which makes use of the SD data is $S_{4}=$ $\sum_{i} S_{i}\left(\frac{r_{i}}{1000 m}\right)^{4} . S_{4}$ separates the primary particle type by the steepness of the LDF, thus leading to smaller $S_{4}$ values for photon showers. Contrary to $L_{L D F}, S_{4}$ is not normalized to the number of triggered stations. Instead, the number of stations $N$ is taken as an additional discriminating photon observable as input for a boosted decision tree (BDT) classifier (Fig. 2). The BDT is trained on two sets of simulated photon and proton showers The final photon candidate cut is placed on the median of the photon distribution of BDT response value such that every event with a larger response is considered a photon candidate. This cut has been defined individually for photon energies above 1, 2, 3, 5 and $10 \mathrm{EeV}$. The application of the trained BDT to data collected between January 2005 and December 2013 resulted in three photon candidates in the first energy range and no photon candidates at higher energies. The upper limits that were placed on the integral photon flux consequently are shown next to the limits from the SD analysis in Fig. 3. The shaded regions around the upper limits were derived by quantifying various systematic effects like changes in the 
hadronic interaction models and uncertainties on the spectral index. Limits on the fraction of UHE photons with respect to the overall cosmic ray flux are also given in [4].
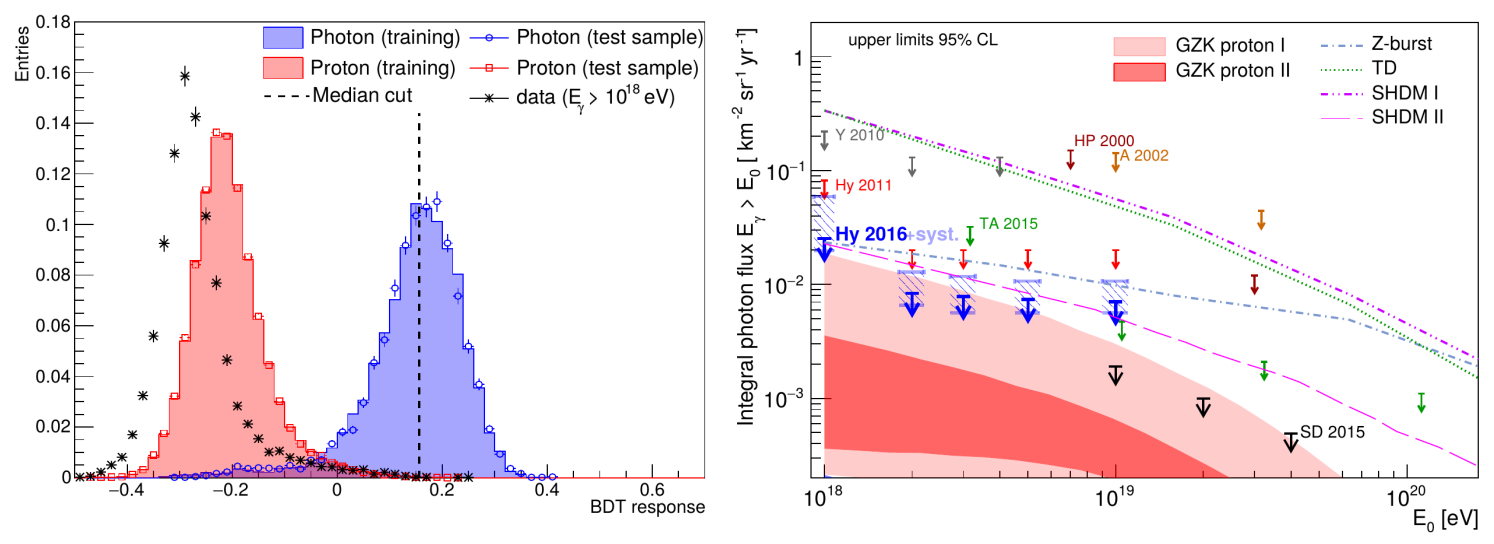

Figure 2: The discriminating parameter in the Figure 3: The upper limits at 95\% C.L. on the insearch for a diffuse UHE photon flux with the hy- tegrated diffuse flux of UHE photons obtained using brid detector is the response of a BDT classifica- the data from the Auger SD (SD 2025) and hybrid detion with the two photon observables $X_{\max }$ and $S_{4}$ tector (Hy 2016) shown together with previous results as input parameters for simulated proton (red) and from Telescope Array (TA)[5], Yakutsk (Y) [6], Havphoton (blue) showers and data (black). The me- erah Park (HP) [7], AGASA (A) [8] and flux predicdian of the photon distribution which serves as the tions from several top-down and cosmogenic photon photon candidate cut is marked by the dashed line. models [9, 10, 11].

\section{Search for point sources of UHE photons}

In order to search for a statistically significant excess of "photon-like" events from point sources [12], at first, a blind search on the full sky has been performed. The energy range of the hybrid detector has been pushed down to $0.2 \mathrm{EeV}$ for this analysis using a BDT with 5 discriminating photon observables among which are $X_{\max }$ and an alternate version of $S_{4}$. Details on the observables can found in [12]. The cut value on the BDT response has been optimized such that the sensitivity is maximized while minimizing the expected upper limits. The background as a function of arrival direction has been estimated using the scrambling technique [13]. The analysis has been applied to data taken between January 2005 and September 2011 by pixelizing the sky into a map with 526,200 target centers. Out of all target centers, the lowest observed $p$-value for the number of "photon like" events was $4.5 \times 10^{-6}$ which corresponds to a chance probability of $36 \%$ to observe that value anywhere in the sky. Therefore no point source of UHE photons could be identified in this analysis and upper limits on the directional photon flux were placed (Fig. 4).

To reduce the penalty factor from an all-sky search in a second approach [14], 364 astrophysical targets have been grouped into 12 target classes, one of them the galactic center. Again, no statistical excess of "photon like" events has been found. The upper limits that were placed on the direction of the galactic center region were compared to an extrapolation of the gamma-ray flux measurements in $\mathrm{TeV}$ range by H.E.S.S.. As a result, part of the phase space for a possible UHE photon flux following a simple extrapolation of the H.E.S.S. data could be constrained. 


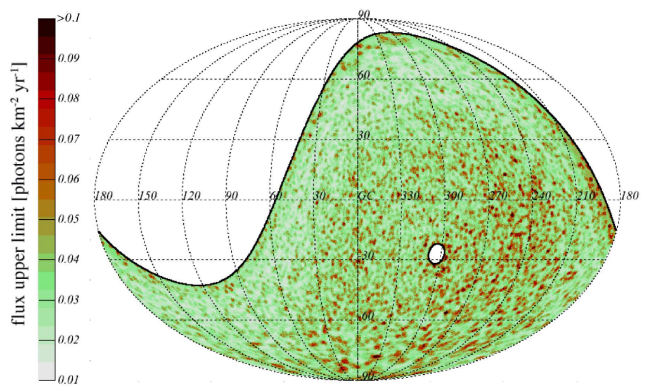

Figure 4: The upper limits at 95\% C.L. on the directional UHE photon flux in galactic coordinates obtained in a blind all-sky search.

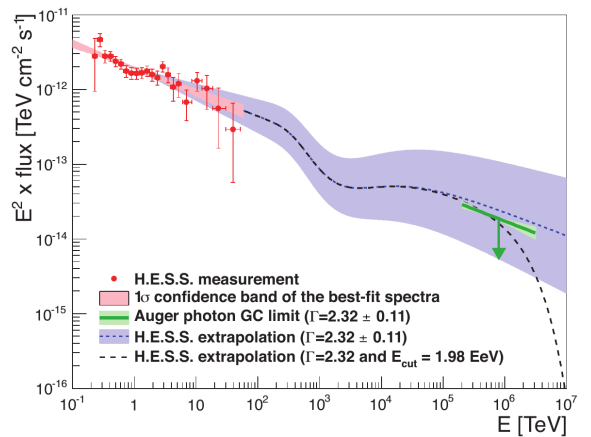

Figure 5: The upper limits on the photons flux from the galactic center region as measured by Auger (green line) together with data measured by H.E.S.S. (red). The light green and blue bands indicate the variation of the assumed spectral index within the uncertainties of the H.E.S.S. measurement. The dashed lines mark the mean of the H.E.S.S. extrapolation with and without a cutoff energy.

\section{References}

[1] The Pierre Auger Collaboration, The Pierre Auger Cosmic Ray Observatory, NIM A 798 (2015) 172.

[2] The Pierre Auger Collaboration, Update of the neutrino and photon limits from the Pierre Auger Observatory, Proc. of 34rd ICRC, The Hague, The Netherlands (2015), PoS (ICRC2015) 1103.

[3] P. Homola et al, Characteristics of geomagnetic cascading of ultra-high energy photons at the southern and northern sites of the Pierre Auger Observatory, Astropart. Phys. 27 (2007) 174.

[4] The Pierre Auger Collaboration, Search for photons with energies above $10^{18} \mathrm{eV}$ using the hybrid detector of the Pierre Auger Observatory, JCAP (2017) 009.

[5] The Telescope Array Collaboration, Constraints on the diffuse photon flux with energies above $10^{18} \mathrm{eV}$ using the surface detector of the Telescope Array experiment, Phys. Rev. D 88 (2013) 112005.

[6] The Yakutsk Collaboration, Constraints on the flux of primary cosmic-ray photons at energies $E>10^{18} \mathrm{eV}$ from Yakutsk muon data, Phys. Rev. D. 82 (2010) 041101.

[7] M. Ave et al, New constraints from Haverah Park data on the photon and iron fluxes of UHE cosmic rays, Phys. Rev. Lett. 85 (2000) 2244.

[8] The AGASA Collaboration, Upper limit on the gamma-ray flux above $10^{19} \mathrm{eV}$ Estimated by the Akeno Giant Air Shower Experiment, Astrophys. J. Lett. 571 (2002) L117.

[9] G. Gelmini et al, GZK Photons as Ultra High Energy Cosmic Rays, JETP. 106 (2008) 1061.

[10] B. Sarkar et. al, Ultra-High Energy Photon and Neutrino Fluxes in Realistic Astrophysical Scenarios Proc. of 32nd ICRC, Beijing, China (2011) Vol. 2, 198.

[11] J. Elis et al, UHECR Particle Spectra from Crypton Decays, Phys. Rev. D 74 (2006) 115003.

[12] The Pierre Auger Collaboration, A search for point sources of EeV photons, Astrophys. J. 789160.

[13] G. Cassiday et al, Mapping the U.H.E. sky in search of point sources, Nucl. Phys. B 14 (1990) 291.

[14] The Pierre Auger Collaboration, A targeted search for point sources of EeV photons with the Pierre Auger Observatory, Astrophsys. J. Lett. 837 (2017) L25. 